\title{
Treatment compliance and risk and protective factors for suicide ideation to completed suicide in adolescents: a systematic review
}

\author{
Natalia C. Rufino, ${ }^{1}$ iD Thiago M. Fidalgo, ${ }^{1}$ (iD Juliana P. dos Santos, ${ }^{1}$ iD Vítor S. Tardelli, ${ }^{1}$ \\ Marcelo G. Lima, ${ }^{1}$ iD Luana P. Frick, ${ }^{1}$ Bojan Mirkovic, ${ }^{2}$ Dartiu X. da Silveira, ${ }^{1}{ }^{*}$ David Cohen $^{3 *}$ \\ ${ }^{1}$ Universidade Federal de São Paulo, São Paulo, SP, Brazil. ${ }^{2}$ University Hospital Charles Nicolle, Centre Hospitalier du Rouvray, Normandie \\ Université, Rouen, France. ${ }^{3}$ Service de Psychiatrie del'Enfant et del'Adolescent, Groupe Hospitalier Pitié-Salpêtriére, Assistance Publique - \\ Hôpitaux de Paris et Université Pierre et Marie Curie, Paris, France. * These authors have contributed equally to this manuscript (senior \\ authorship).
}

\begin{abstract}
Objective: To review the literature regarding adolescent suicide risk and explore the associations between treatment compliance (expressed as a concept including measured adherence to treatment and/or mental health service utilization) and risk and protective factors for suicidal behavior (SB), as well as the association between treatment compliance and reattempts.

Methods: PubMed, LILACS, and Google Scholar were searched using the following terms:

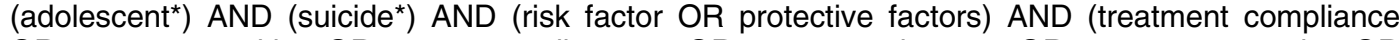
OR treatment attrition OR treatment adherence OR treatment drop out OR treatment retention OR mental health utilization). We retrieved studies that focused on the relation of treatment compliance to risk and protective factors for SB and that had only adolescent samples.

Results: Of 4,841 articles, 30 original articles were selected for review. Most studies indicated high mental health service (MHS) utilization and poor treatment adherence by SB patients. Social minority status and conduct disorder were associated with less treatment adherence, while female sex, parental perceived need for treatment, and major depression were associated with greater treatment adherence. Inpatient and intensive emergency care after SA and family interventions improved MHS utilization and treatment compliance. However, we found no substantial protective effect of treatment compliance against reattempts.

Conclusion: Effective treatment planning for compliance requires considering psychopathology, treatment planning, and social, familial, and individual factors.
\end{abstract}

Keywords: Suicidal behavior; adolescent; reattempts; treatment compliance

\section{Introduction}

Suicide, which currently accounts for $6 \%$ of deaths in young people worldwide, is the second leading cause of mortality among girls and the third among boys aged 15 to 24 years. ${ }^{1}$ In that context, risk-taking behaviors, including suicidal behavior (SB), are among the most serious threats to the health and safety of adolescents and young adults. ${ }^{2-4}$

Epidemiological data indicate an increase in the prevalence of $\mathrm{SB}$ in recent years, especially among girls and migrants. ${ }^{5,6}$ SB may be represented in a spectrum of increasing severity, from suicidal ideation (SI) to suicide attempt (SA) to completed suicide (CS). ${ }^{7}$ Prevalence patterns run in the opposite direction, with studies showing $\mathrm{SI}$ as the most prevalent, followed by SA and CS. ${ }^{8}$

Adolescent SAs are a public health problem associated with high morbidity, high mortality, and negative

Correspondence: Natalia C. Rufino, Rua Major Maragliano, 241, CEP 04017-030, São Paulo, SP, Brazil.

E-mail: natrufino@ hotmail.com

Submitted Apr 12 2020, accepted Sep 09 2020, Epub Jan 292021. psychosocial implications. ${ }^{9,10}$ In the United States, a large nationwide study revealed that between 2007 and 2015, emergency department (ED) visits for SB doubled among youth. ${ }^{11}$ This raises major concerns, given the evidence showing previous SA in one-third of adolescents who die from suicide ${ }^{12}$; in fact, it is well established that previous $\mathrm{SA}$ is the strongest predictor of subsequent death by suicide. $^{13}$

Nearly $90 \%$ of adolescents who commit suicide have a psychiatric disorder, and more than $60 \%$ of young people are depressed at the time of death. ${ }^{13}$ Thus, to lower the risk of suicide, it is essential to treat underlying comorbidities. ${ }^{14}$ However, poor treatment compliance emerges as an important obstacle among adolescents with SB, with studies highlighting the initial phases of treatment as the most vulnerable period. ${ }^{15,16}$ Longitudinal studies have identified treatment attrition as an important marker of
How to cite this article: Rufino NC, Fidalgo TM, dos Santos JP, Tardelli VS, Lima MG, Frick LP. Treatment compliance and risk and protective factors for suicide ideation to completed suicide in adolescents: a systematic review. Braz J Psychiatry. 2021;43:550558. http://dx.doi.org/10.1590/1516-4446-2020-1026 
suicide reattempts, ${ }^{17,18}$ whereas treatment compliance has been recognized as a protective factor for suicidality. ${ }^{19,20}$

The definition of compliance varies widely. ${ }^{21,22}$ In the present article, compliance will be understood as a complex phenomenon that reflects the patient's contribution to the management of his or her own treatment, ${ }^{21}$ including the capacity to enter treatment, implement the instructions delivered, and follow through to treatment completion, as well as the ability of the therapist to negotiate and motivate the patient regarding treatment ${ }^{21}$ and the reduction of social barriers to entering treatment. ${ }^{23}$ Factors associated with increased treatment compliance among suicidal adolescents include parental involvement in treatment, ${ }^{18}$ while factors associated with decreased compliance include depressive disorders, ${ }^{24}$ substance abuse, ${ }^{25}$ and conduct disorders. ${ }^{25}$

There is still a lack of literature concerning the factors associated with treatment compliance in adolescents with SI and SA, and concerning the role of treatment compliance in preventing reattempts and CS. Information about treatment compliance is fundamental to ensure treatment efficacy and prevent reattempts. Here, we review the literature on adolescents (11 to 18 years old) with SI, SA, or CS. We aimed to explore: i) the association of risk and protective factors with treatment compliance expressed as adherence to treatment or mental health service (MHS) utilization in the context of SI, SA, or CS, and ii) whether treatment compliance was a protective factor for reattempts.

\section{Methods}

We conducted a systematic review of the literature on risk and protective factors for SI, SA, and CS among adolescents, with a focus on the relationships between these factors and treatment compliance. Details of the present systematic review protocol were registered with PROSPERO and can be accessed at https://www.crd. york.ac.uk/PROSPERO/display_record.php?RecordID= 98701.

Because the definition of compliance can be expressed with different words, we extended the search to several possible synonyms (adherence, attrition, retention, MHS utilization, and drop out). We searched PubMed, LILACS, and Google Scholar for publications in English, French, Spanish, and Portuguese (the languages in which the authors are fluent) from January 1988 until June 2020. The following search terms were used: (adolescent ${ }^{*}$ ) AND (suicide $^{\star}$ ) AND (risk factor OR protective factors) AND (treatment compliance OR treatment attrition OR treatment adherence OR treatment drop out OR treatment retention OR mental health utilization). This search strategy was adapted for each database to maximize the search results (the complete search strategy for each database is available as online-only supplementary material). The screening and extraction were performed in duplicate by two independent authors (NCR and JPS).

We included studies regarding SB (SI, SA, CS, AND/ OR reattempts) that: i) reported on adolescent samples (11-18 years); ii) addressed the subject of risk and protective factors; and iii) discussed the relation between SB and treatment compliance.
The initial search retrieved 4,841 publications from the three databases. At the title-screening stage, 4,161 articles were excluded due to incompatibility with the aims of the review and 680 continued to the abstract-screening stage. Four case reports, seven duplicates, and 480 studies were excluded because they did not fulfil the inclusion criteria, and the full text of 79 articles was examined. Of these, 49 publications that did not focus on treatment compliance in adolescents with SB and were excluded. Our final sample consisted of 30 articles. Figure 1 shows the flowchart of publication selection. We extracted the following information from the final sample: 1) author/year; 2) country where the study was conducted; 3 ) sample size; 4) age range of the included patients; 5) study design; 6) time of follow-up; 7) sample details; 8) main outcome; 9) compliance findings; 10) risk or protective factors for SI, SA, and CS; and 11) limitations.

The selected articles were grouped for analysis according to their focus: articles reporting on MHS utilization; or articles in which MHS utilization was assessed in a specific sample, for a specific time, without measurement of attendance frequency, or in terms of the need for services. ${ }^{26}$ Adherence to treatment was considered when the study measured the frequency with which patients attended services (as the terminology of noncompliance was defined by each publication). ${ }^{21}$

\section{Results}

\section{Description of the studies}

We assessed eight cross-sectional studies ${ }^{27-34}$ and 22 longitudinal studies ${ }^{25,35-55}$ (four of which were interventional studies, ${ }^{42,43,53,55}$ including two randomized controlled trials). ${ }^{42,53}$ The longitudinal studies varied widely in length of follow-up, from 1 month $^{53}$ to 9 years. ${ }^{41}$ Most studies $(21$ of $31 ; 67 \%$ ) were conducted in the United

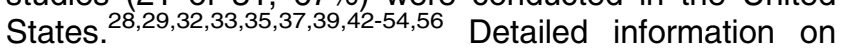
the 30 studies is shown in Table $\mathbf{S 1}$. Table 1 summarizes the factors related to adherence to treatment, MHS utilization, and risk and protective factors for SI, SA, and CS. The results from the 79 preliminarily articles selected for full text analysis are presented in Tables S2-S4, available as online-only supplementary material.

\section{Treatment compliance}

Because some studies described MHS utilization without systematically measuring the length of treatment before drop-out, findings were summarized according to the following topics: i) MHS utilization and associated factors; ii) adherence to treatment and associated factors (in the presence of a systematic measure of treatment completion); and iii) relationship of MHS utilization and adherence with reattempt rates.

\section{Mental health service utilization and associated factors}

MHS utilization varied from $29^{28}$ to $86.2 \%{ }^{29}$ among adolescents with SB (including SA, SI, and CS). 


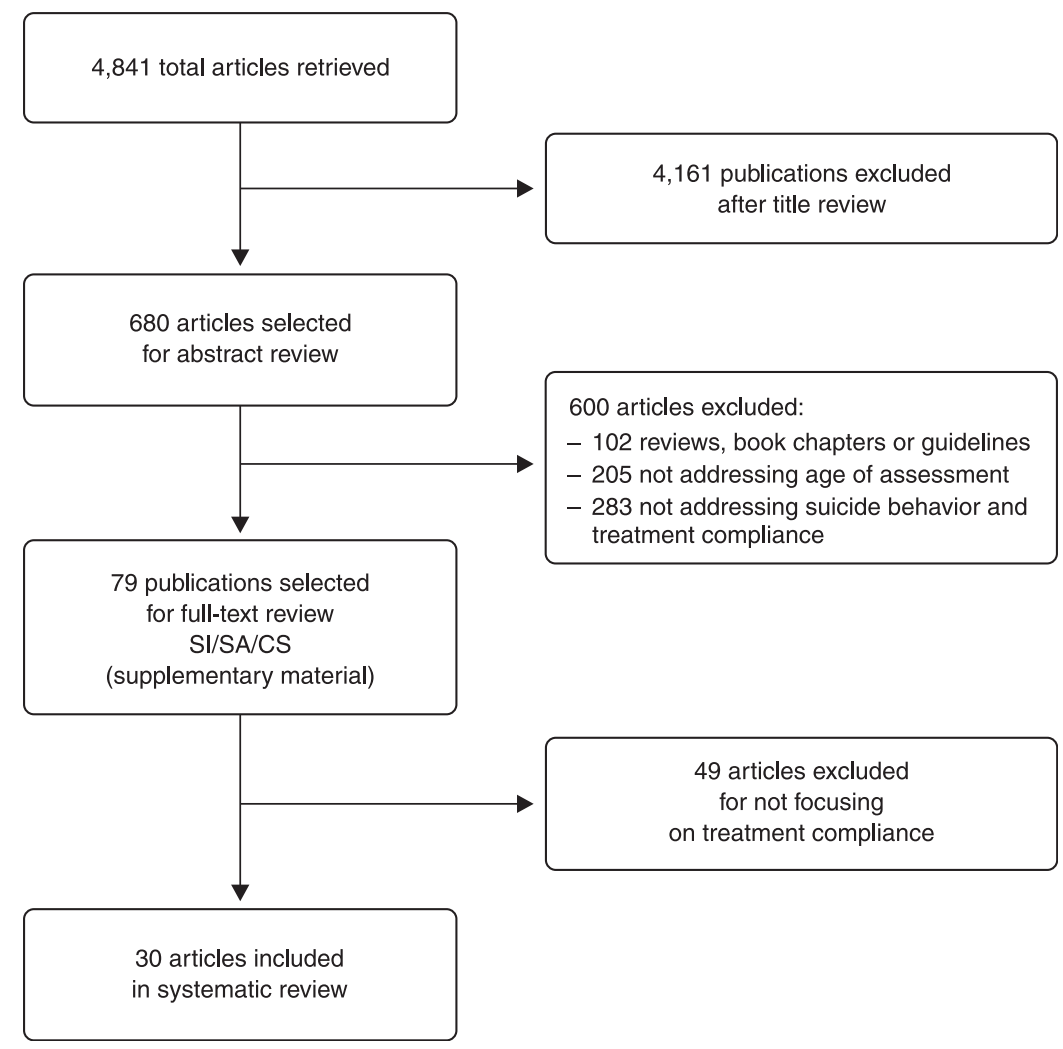

Figure 1 Flowchart of article selection. $\mathrm{CS}=$ completed suicide; $\mathrm{SA}=$ suicide attempt; $\mathrm{SI}=$ suicide ideation.

The rate of MHS utilization among adolescents with SB differed across four studies, ${ }^{27-29,33}$ In a cross-sectional study with a sample of 10,148 adolescents, Nock et al. ${ }^{29}$ found that 66.4 to $86.2 \%$ of the adolescents used some form of MHS. In turn, Freedenthal ${ }^{28}$ found that only $29.07 \%$ of 2,226 patients interviewed in a household survey who reported SB had received mental health treatment in the past year. In a sample of 948 patients, Wu et al. ${ }^{33}$ found a $45 \%$ MHS utilization rate, of which $59 \%$ were outpatient, $22 \%$ were inpatient, and $19 \%$ were school-based. The prevalence rates of lifetime service utilization among adolescents with SB in the Mexican Adolescent Mental Health Survey ${ }^{27}$ was $35 \%$ for those with SI, $44 \%$ for those with a suicide plan, and $50 \%$ for those with SA; the prevalence rates of 12-month service utilization dropped dramatically to 10,24 and $21 \%$, respectively.

In contrast, studies with clinical samples of adolescents with SB reported high rates of service utilization among participants. Kataoka et al., ${ }^{44}$ in a 5-month follow-up of 95 adolescents with SB, found that $71 \%$ of the sample received some sort of treatment, ${ }^{44}$ similar to the $79 \%$ reported by Yen et al. ${ }^{54}$ in a sample of 99 adolescents after hospitalization due to SB. O'Mara, ${ }^{47}$ in a sample of 96 adolescents, found a service utilization rate (psychotherapy, psychiatric medications, psychiatric hospitalization, and/or drug and alcohol treatment) after hospitalization for SB of $81 \%$ in a 6-year follow-up. Groholt \& Ekeberg ${ }^{41}$ examined the prevalence of SB 8 to 10 years after SA in a sample of 71 hospitalized adolescents. Despite low compliance shortly after the index SA, suicide attempters received a substantial amount of treatment during follow-up. ${ }^{41}$ Finally, the only study on CS found that the sample of 55 adolescents who committed suicide had significantly more current and lifetime experience with mental health professionals (including psychiatrists) and youth protection services than healthy controls, which probably reflected present and severe lifetime psychiatric symptoms. ${ }^{30}$

Among all the publications reviewed, those that evaluated factors associated with MHS utilization found that being male, ${ }^{30,43,54}$ older ${ }^{48}$ Latino, ${ }^{28,31,33,44}$ or identified as a racial minority, ${ }^{28,31,57}$ and being a first- or second-generation migrant ${ }^{31,33,57}$ were all associated with less MHS utilization. In one study, ${ }^{33}$ poverty was not associated with less access to services, but was associated with a lower possibility of receiving specialized care. However, Wu et al..$^{33}$ found that access to Medicaid or Medicare was associated with higher service utilization among adolescents with SB in the United States.

Past $\mathrm{MHT},{ }^{48}$ presenting with severe depressive ${ }^{43,44}$ or other psychiatric ${ }^{28}$ symptoms, such as anxiety ${ }^{33}$ or disruptive behaviors, ${ }^{33}$ or a diagnosis of substance use disorder ${ }^{33}$ were all related to higher service utilization in several studies. In a sample of suicide completers, Renaud et al. ${ }^{30}$ found that subjects with depressive and anxiety disorders had received more psychiatric and general MHT in the year before committing suicide, and those who had been hospitalized within the month before committing suicide were more likely to have abused alcohol and experienced psychosis.

Having a history of $\mathrm{SA}^{43,44,48}$ was associated with increased service utilization, including outpatient treatment ${ }^{48}$ 
Table 1 Factors related to treatment compliance, MHS utilization, and risk and protective factors for SI, SA, and CS

\begin{tabular}{|c|c|c|c|c|c|c|c|c|c|}
\hline & \multirow[b]{2}{*}{ MHSU } & \multirow[b]{2}{*}{ ITC } & \multirow[b]{2}{*}{ DTC } & \multirow[b]{2}{*}{ RSA } & \multicolumn{3}{|c|}{ Risk factors } & \multicolumn{2}{|c|}{ Protective factors } \\
\hline & & & & & SI & SA & CS & SI & SA \\
\hline \multicolumn{10}{|l|}{ Cultural and sociodemographic factors } \\
\hline Gender (female) & $\uparrow X$ & & $\mathrm{X}$ & $x$ & $\mathrm{X}$ & $\mathrm{X}$ & & & \\
\hline Minority status (migration, ethnicity) & $\downarrow x$ & & $\mathrm{x}$ & $\mathrm{X}$ & $\mathrm{X}$ & $X$ & & & \\
\hline Age (older) & $\downarrow x$ & & & & & & & & \\
\hline Age (younger) & & & & & & $\mathrm{X}$ & & & \\
\hline \multicolumn{10}{|l|}{ Family factors } \\
\hline Parental perceptions of treatment as helpful & $\uparrow X$ & $x$ & & & & & & & \\
\hline Cohesive family relationships, family functioning & & $x$ & $\begin{array}{l}x \\
x\end{array}$ & & & & & $x$ & $\mathrm{x}$ \\
\hline $\begin{array}{l}\text { Family composition (nonintact family) and relationship distress } \\
\text { (maternal hostility, family conflict, abuse) }\end{array}$ & $\uparrow X^{*}$ & $x$ & $\begin{array}{l}x \\
x\end{array}$ & & $x$ & $x$ & $\mathrm{X}$ & & \\
\hline Parental perceived need for services & $\uparrow X$ & & & & & & & & \\
\hline Parents with severe psychiatric disorders & $\uparrow X$ & & & & & & & & \\
\hline $\begin{array}{l}\text { Adolescents doing more activities/spending more time with parents } \\
\text { Personality and cognitive factors }\end{array}$ & $\uparrow X$ & & & & & & & $\mathrm{x}$ & \\
\hline $\begin{array}{l}\text { Disengagement, distractibility, poor school performance/academic } \\
\text { problems, hopelessness }\end{array}$ & & & $\mathrm{X}$ & & $x$ & $\mathrm{X}$ & & & \\
\hline Involvement in physical fighting & & & $\mathrm{X}$ & & & & & & \\
\hline \multicolumn{10}{|l|}{ Health issues } \\
\hline $\begin{array}{l}\text { Posttraumatic stress disorder, major depression disorder, } \\
\text { substance use disorder }\end{array}$ & $\uparrow X$ & $x$ & $X^{\dagger}$ & & $x$ & $x$ & $x$ & & \\
\hline Anxiety disorder & $\uparrow X$ & $\mathrm{X}$ & $\mathrm{X}$ & & $\mathrm{x}$ & & $\mathrm{X}$ & & \\
\hline Bipolar disorder and psychosis & $\uparrow X$ & & & & & & $\mathrm{x}$ & & \\
\hline Personality disorder & $\uparrow X$ & & & & & $\mathrm{X}$ & & & \\
\hline Disruptive behavior disorder & $\downarrow x$ & $\mathrm{X}$ & $X^{\ddagger}$ & & $\mathrm{x}$ & $\mathrm{x}$ & & & \\
\hline Conduct disorder & $\downarrow x$ & & $\mathrm{X}$ & & $\mathrm{x}$ & $\mathrm{x}$ & & & \\
\hline Previous SA & $\uparrow x$ & $\mathrm{X}$ & & & & $x$ & $\mathrm{X}$ & & \\
\hline Previous SI & $\uparrow X$ & & & & $\mathrm{x}$ & $\mathrm{x}$ & & & \\
\hline Length of time planning suicide & $\uparrow X$ & $x$ & & & & & & & \\
\hline Use of psychotropic medications & & $x$ & & & & & & & \\
\hline Psychotherapy & $\downarrow X^{\S}$ & $x$ & & & & & & & \\
\hline Suicide completion & $\uparrow X$ & & $x$ & & & & & & \\
\hline \multicolumn{10}{|l|}{ Types of interventions } \\
\hline Type of hospital care/post-discharge plan & & $\mathrm{X}$ & & & & & & & \\
\hline Past outpatient treatment & $\uparrow X$ & & & & $\mathrm{x}$ & $x$ & & & \\
\hline Inpatient treatment & $\uparrow X$ & $\mathrm{x}$ & & $\mathrm{x}$ & & & & & \\
\hline Intensive emergency care & & $x$ & & & & & & & \\
\hline Family intervention & & $\mathrm{X}$ & & & & & & & \\
\hline
\end{tabular}

CS = completed suicide; DTC = decrease treatment compliance; ITC = increase treatment compliance; MHS = mental health service; MHSU = mental health service utilization; $\mathrm{PD}=$ psychiatric disorders; RSA = repeated suicide attempt; $\mathrm{SA}=$ suicide attempt; $\mathrm{SI}=$ suicide ideation. * Adolescents not living with both parents, with worse self-perceived health, and disruptive behaviors had more hospitalizations.

Adolescents with affective/anxiety disorders were less compliant with psychopharmacology.

$\$$ Adolescents with disruptive behavior disorders were less compliant with psychotherapy.

${ }^{\S}$ Adolescents with more severe psychopathology and conduct disorder were less likely to use psychotherapy.

and school-based services ${ }^{44}$ and more intensive MHS treatment warranted by continued suicide risk. ${ }^{44}$ Inpatient care was associated with not living with both parents, poorer self-perceived health, foreign origin, and disruptive behaviors ${ }^{33}$; intensive care treatment (including inpatient, residential and emergency treatment) was associated with more impaired adolescents, who also tended to make less use of psychotherapy. ${ }^{33}$ Interestingly, adolescents whose parents had mood disorders were more likely to receive outpatient treatment and less likely to receive intensive treatment. $^{54}$

With regards to psychotherapy, patients with borderline personality disorder were more likely to participate in group therapy, and patients with anxiety disorder were more likely to attend individual psychotherapy, ${ }^{54}$ while a diagnosis of conduct disorder was associated with less frequent use of psychotherapy. ${ }^{54}$

Finally, parental perceptions of the need for $\mathrm{MHT}^{31}$ and participating in more activities with parents ${ }^{34}$ were asso- ciated with greater MHS utilization, with the latter also being a protective factor for $\mathrm{SI}^{34}$

\section{Adherence to treatment}

The reported rates of treatment adherence ranged from $32^{25}$ to $96 \%{ }^{36}$ The studies used different follow-up periods and different criteria to define adherence.

Seven studies found adherence levels below $50 \%{ }^{25,37,40-42,52,56}$ Groholt \& Ekeberg ${ }^{41}$ found that $41 \%$ of participants attended three or more psychotherapy sessions after the index SA in a sample of 71 hospitalized adolescents. Spirito et al., ${ }^{56}$ studying 62 adolescents after $\mathrm{SA}$, found that $48 \%$ remained in treatment until the end of the 3-month follow-up. Giraud et al., ${ }^{40}$ in a sample of 517 suicide attempters, reported that $35 \%$ were optimally observant of the care proposed by the attending clinician. In an 18-month follow-up study with 115 teenagers, Trautman et al. ${ }^{52}$ found that adherence to treatment before 
dropout was significantly shorter for attempters than for nonattempters. Granboulan et al. ${ }^{25}$ followed 163 adolescents for 3 months after hospitalization due to SB and found that $25.5 \%$ never attended any follow-up visits; only $32 \%$ showed up for all scheduled appointments. Burns et al. ${ }^{37}$ assessed 85 adolescents over 2 years after hospitalization due to SA and found that $57 \%$ were noncompliant with psychotherapy, and that $41.3 \%$ were noncompliant with medication in at least one 6-month follow-up assessment. ${ }^{37}$ Finally, Grupp-Phelan et al. ${ }^{42}$ found that 15 to $54 \%$ of 24 adolescents who presented to the ED with suicide risk attended MHS, depending on whether they were from the control or the intervention group.

In contrast, five studies reported higher treatment adherence. ${ }^{36,39,45,46,51}$ In a sample of 100 discharged adolescents with $\mathrm{SB}^{45,46}$ followed for 6 months, initial adherence was found to be highest for individual therapy (90\% of adolescents attended two or more sessions) and lowest for parent guidance/family therapy (65\% attended more than one session) and when medication was recommended $(76 \%$ of the adolescents attended more than one appointment). Burgess et al., ${ }^{36}$ in a sample of 25 individuals with SA, found a very high adherence rate (96\%) in a 3-month follow-up study. Czyz et al. ${ }^{39}$ evaluated adherence with a month-long daily electronic remote-based intervention conducted with 34 post-discharge teenagers who had been hospitalized due to SA and found an overall adherence rate of $69 \%$.

\section{Factors associated with adherence to treatment}

In a study comparing a problem-solving ED-intervention with a treatment-as-usual control group, Spirito et al. ${ }^{51}$ reported an overall adherence of $74.2 \%$, with the intervention group attending an average of 8.4 sessions and the control group attending an average of 5.8 sessions. Czyz et al. ${ }^{39}$ found an overall adherence rate of $69 \%$ to a month-long, daily, electronic, remote-based intervention conducted with 34 post-discharge teenagers who had been hospitalized due to SA. The researchers also found that adherence among patients with SI was half that among suicide attempters.

Alcohol use at the time of the attempt, ${ }^{56}$ higher SI scores on structured measures, ${ }^{49}$ the length of time spent planning the suicide, ${ }^{56}$ worse depression and anxiety scores at the initial assessment, ${ }^{25}$ impulsivity, ${ }^{49}$ illicit drug ${ }^{25}$ use, and premeditated $\mathrm{SA}^{25}$ as well as severe psychopathology, ${ }^{25}$ were all associated with greater adherence. Adolescents with worse scores on measures of depression, hopelessness, anger, drinking, suicide intent, and family functioning were more likely to be prescribed psychotropic medications, and adolescents taking psychotropic medications attended more appointments than those not taking medication. ${ }^{51,56}$ Finally, Czyz et al. ${ }^{39}$ found that a previous SA was the only factor associated with survey adherence.

Being female, ${ }^{52}$ having more severe child psychopathology ${ }^{37}$ or disruptive behavior disorder, ${ }^{50}$ and being frequently involved in physical altercations ${ }^{56}$ ' were all related to nonadherence to treatment in general, as were maternal psychopathology, ${ }^{46}$ having a family member with a health problem, ${ }^{50,56}$ and cohesive or adaptive family relationships. ${ }^{49,50}$ Having a mother less adherent to treatment was a risk factor for $\mathrm{SI}$ in a sample of 65 sexuallyabused children and adolescents. ${ }^{32}$

Disruptive behavior disorders, ${ }^{37}$ substance use disorders other than alcohol use disorder, ${ }^{37}$ and anxiety ${ }^{50}$ were all related to worse adherence to psychotherapy, while family dysfunction and poor father-offspring relationships were related to worse adherence to family therapy. ${ }^{46}$ Mood and anxiety diagnoses, as well as worse fatheroffspring relationships, ${ }^{46}$ were found to be related to worse adherence to medication. ${ }^{37}$

Parental perceptions of the helpfulness of treatment ${ }^{37}$ and living in single-parent households ${ }^{49,50}$ predicted increased adherence, just like initial inpatient care $^{25,51}$ and specialized ED interventions. ${ }^{42,49,50,53}$ Granboulan et al. ${ }^{25}$ found that, in a sample of 163 adolescents, those who were hospitalized longer after their SAs had received inpatient individual and family therapy sessions, which may have helped improve adherence to outpatient treatment. That study also found that adherence was better when hospital staff scheduled appointments with the therapist to whom they were referring adolescents than when patients and families scheduled the appointments themselves. ${ }^{25} \mathrm{~A}$ specialized emergency room program significantly reduced treatment-resistant attitudes by the mother, leading to higher treatment adherence in a sample of 140 adolescents who visited an ED after SA in a 6-month follow-up study, ${ }^{49}$ and slightly higher adherence compared to that of the standard care group in an 18-month follow-up study. ${ }^{50}$ TeenScreen-ED (a program consisting of motivational interviews, barrier reduction, outpatient appointments, and reminders before scheduled appointments) improved adherence to outpatient treatment in patients with SB presenting at pediatric EDs. ${ }^{42}$ Similarly, patients who participated in a family-based crisis intervention program were significantly less likely to be hospitalized than patients who underwent treatment as usual in a sample of 139 adolescents who visited EDs. ${ }^{53}$

Finally, a study comparing a problem-solving intervention in an ED and a treatment-as-usual control group ${ }^{51}$ reported an overall adherence of $74.2 \%$, with the intervention group attending on average 8.4 sessions vs. 5.8 sessions in the control group.

Relationship of mental health service utilization and adherence with reattempt rates

Several studies examined the relationship between repeated SA and treatment compliance or MHS utilization. However, in most studies, there was no evidence of treatment compliance being a protective factor for repeated SA.

Yen et al. ${ }^{54}$ examined treatment utilization in a sample of 99 adolescents previously hospitalized due to risk of suicide and followed for 6 months. Even with high rates of MHS utilization, repeated SA rates remained high (19\%). ${ }^{54}$ Another study found low treatment compliance in a 1-year follow-up, with only $35 \%$ of patients adhering to the care proposed and a $15 \%$ prevalence of hospital referrals due to a repeated SA. ${ }^{40}$ 
In a 1-year follow-up, Normand et al. ${ }^{57}$ found that $13.3 \%$ of their sample reattempted suicide. Of those, $65.2 \%$ had ongoing psychological care for 1 week, ${ }^{56.5} \%$ had ongoing psychological care for 1 month, and $34 \%$ had ongoing psychological care for 6 months to 1 year. ${ }^{57}$

Higher percentages of repeated SA were observed by Groholt \& Ekeberg ${ }^{41}$ in a longer follow-up of 8 to 10 years: $44 \%$ of their sample reattempted suicide. However, importantly, half of that sample had a mood or personality disorder diagnosis, both of which were associated with higher rates of repeated $\mathrm{SA}$ in the study. ${ }^{41}$

Two studies did not find a significant difference in treatment compliance between adolescents with or without repeated SA. ${ }^{37,45}$ Additionally, another study found that despite the availability of treatment and the high levels of compliance and satisfaction with treatment, 3 months after SA a large proportion of adolescents considered overdosing again if they found themselves experiencing similar difficulties. ${ }^{36}$

\section{Discussion}

The aim of this study was to review the literature concerning risk and protective factors for SI, SA, and $\mathrm{CS}$ and their relationship with treatment compliance in patients with SB. To the best of our knowledge, this is the first systematic review of risk and protective factors for SB and of the relationship between risk factors and treatment compliance as well as MHS utilization by adolescents. Three substantial findings emerge from this review: i) clinical samples of adolescents with SB are likely to use MHS frequently, however with poor treatment adherence; ii) family involvement and ED visits or initial inpatient care interventions are related to increased MHS utilization and treatment compliance; and iii) there is no significant relationship between treatment compliance or $\mathrm{MHS}$ utilization and reattempts.

The literature on treatment compliance/adherence varies widely in terms of methodology and measures and with respect to the diseases, patients, and treatment regimens studied. Therefore, differences in measurement and context produce wide variations in adherence estimates, correlates, and outcomes. ${ }^{13,16,49,58,59}$ This issue was addressed in the present study, in which the manner of treatment compliance/adherence measurement was considered, with results reported in terms of $\mathrm{MHS}$ utilization and treatment compliance according to the definitions presented in each article.

The articles addressing MHS utilization in this review reported different levels of utilization intensity and a variety of interventions (ED care, specialized outpatient clinics, and inpatient hospital treatment) among adolescents with SB. This finding is consistent with observations in clinical practice and in the literature. ${ }^{60}$ Importantly, despite poor treatment compliance immediately after the emergence of SB, patients with $\mathrm{SB}$ are described as frequent users of $\mathrm{MHS}{ }^{41} \mathrm{~A}$ case-control study comparing 129 young people with serious SA and 153 controls $^{60}$ found a high percentage of lifetime MHS utilization among patients with SA (78.3\%), but fewer contacts with services in the month preceding the SA (58.9\%), which may point to lower adherence rates close to SA.

Being a woman was associated with higher lifetime MHS utilization. ${ }^{30,43,54}$ This association may support the idea that females are more open to talking about their mental health issues and to seeking help. ${ }^{61-64}$ Conversely, being a member of a racial minority ${ }^{31,44,57}$ was predictive of receiving less treatment. This finding is probably associated with the cultural and socioeconomic barriers faced by such populations, which are related to an increased risk of SI and SA. ${ }^{65}$ Therefore, interventions designed to primarily address family, individual and cultural barriers to treatment should target increased MHS utilization. $^{51,66}$

Several mental health factors were found to be related to increased MHS utilization: depression, anxiety disorder, borderline personality disorder, and history of a prior SA. ${ }^{30,44,54}$ Considering the financial cost of erratic MHS utilization, ${ }^{7,67-69}$ strategies aiming to improve the initial use of services and stratify service delivery according to disorder may entail better resource distribution in suicide prevention. For instance, we found that patients with borderline personality disorder were more likely to use group therapy interventions, while those with anxiety disorders preferred individual psychotherapy. ${ }^{54}$ As expected, patients with conduct disorders had poor adherence to outpatient treatment, which may be related to the necessity of more restrictive care offered by residential treatment. ${ }^{54}$

We know from the literature that treatment with evidence of effectiveness for conduct disorder requires resource-intensive approaches and substantial parental involvement. Therefore, the families most in need of treatment may be the least likely to obtain and adhere to it. Conduct disorder comorbidity may render affected youth less attractive to treating clinicians and more disruptive in treatment settings not specifically geared to address conduct disorder. This should be further investigated in studies with standard measures for psychiatric disorders and treatment compliance, which will improve the current understanding regarding development of public policies to improve the cost-effectiveness of treatments. Interventions that involve young people in the development and delivery of services ${ }^{70}$ and that consider their preferences, barriers, and cultural characteristics might favorably impact service utilization by adolescents, especially those with suicidal risk behavior. ${ }^{70}$

It is clear that interventions are also affected by socioeconomic and political factors. In the United States (where two-thirds of the reviewed studies were performed), there is a legal requirement to evaluate and ensure the medical stability of all patients who present at the ED, regardless of the patient's ability to pay. However, the ability to access follow-up care, including MHT, varies widely as a function of insurance coverage, representing a potentially enormous barrier to treatment. Conversely, the structure of the health care system may have an impact on treatment compliance. In our review, one study found that having insurance (Medicaid or Medicare) increased MHS utilization, ${ }^{33}$ whereas another found no impact of poverty on access to treatment, ${ }^{43}$ suggesting that individuals with lower socioeconomic status tended to 
be cared for more often by general practitioners than by mental health specialists. This sheds light on the importance of in-depth analysis of this relationship in future studies.

Family factors, as well as engaging in more activities and spending more time with parents, were related to increased MHS utilization; spending more time with parents was also a protective factor for $\mathrm{SI}^{34}$ This finding is important for clinicians because, despite the need to promote autonomy in adolescence, the presence of parents remains very important, either to protect against SB or to obtain treatment for it. A controversial family factor shown by Rotherdam-Borus et al. ${ }^{49}$ was that more cohesive and adaptive families tended to comply less with treatment. The authors suggest that more cohesive families may show faster improvements and restructuring of relationship patterns, which may explain the high number of dropouts. However, this is an issue that will require further studies.

Regarding treatment compliance, the majority of studies evaluated patients after hospitalization or after ED evaluation, $25,36,37,39-42,45,47,50,51,53-57$ which may select for better compliance levels since the data collected showed that inpatient care is a protective factor for compliance..$^{25,51}$ However, most (seven) studies ${ }^{25,37,40-42,52,56}$ reported low levels of compliance with outpatient treatment. Among the four studies that found good compliance, one study evaluated attendance to one or two appointments after hospitalization, ${ }^{45}$ another study evaluated only 25 adolescents with $A S,{ }^{36}$ one was an interventional study, ${ }^{51}$ and one study evaluated compliance with a month-long, daily, electronic, and remote-based intervention rather than a particular outpatient treatment. ${ }^{39}$ Therefore, the most robust evidence was for low treatment compliance. The findings of Renaud et al. ${ }^{30}$ that $54.4 \%$ of suicide completers received treatment but were poorly compliant or noncompliant corroborates previous studies suggesting that noncompliance with recommended aftercare is particularly common among adolescents with SB. ${ }^{41,71}$

As found for MHS utilization, adolescents with more severe psychopathology were more compliant with treatment, ${ }^{25,51,56}$ possibly because of the level of care warranted. Additionally, prescription of psychotropic medications was associated with an increase in compliance. ${ }^{51}$ We also noted that adolescents whose parents perceived treatment to be helpful and adolescents whose parents were diagnosed with a mood disorder were more likely to attend outpatient treatment. In the latter situation, a parent's mental health condition could lead to the recognition of the importance of outpatient treatment. ${ }^{54}$ Findings reported by Jon-Ubabuco \& Dimmitt Champion ${ }^{66}$ and Bushnell et al. ${ }^{72}$ corroborate that hypothesis. The first study found that African American caregivers who struggled with mental health issues themselves were better able to recognize these issues in adolescents and were more predisposed to seek out MHS. Bushnell et al. expanded the scope of the issue to how parents understood their overall health (by assessing their adherence to statin and antihypertension medication use) and the effect this had on adherence to their children's anxiety treatment, concluding that perceptions of the parent's own health and need for treatment could be an important predictor of child and adolescent treatment compliance overall. ${ }^{72}$

Interventions occurring in $\mathrm{EDs}^{42,50,53}$ and in initial inpatient treatment ${ }^{25,51}$ were shown to improve treatment compliance. Several other studies have addressed the effects of ED interventions on the treatment compliance of adolescents and young people with SB. Lachal et al. ${ }^{73}$ reinforced the efficacy of enhanced ED interventions for improving MHS compliance. Additionally, they noted that the interventions that seem the most effective are those that target the time both during and after ED discharge, those that are implemented most rapidly after discharge, those that actively include parents, and those that inform families about barriers to follow-up. ${ }^{73}$

Regarding the association between treatment compliance and reattempts, we found no evidence in our review that treatment compliance reduces the number of reattempts over time ${ }^{41,54,57}$ Likewise, we found no significant evidence that treatment compliance can prevent $\mathrm{SI}$ or SA. ${ }^{45}$ However, a matched cohort study with a sample of adults followed for 20 years after an episode of deliberate self-harm in Denmark showed that those receiving psychosocial therapy at a suicide prevention clinic had lower odds of dying due to mental or behavioral alcoholrelated causes than patients receiving standard care. Additionally, this population had reduced odds of dying from suicide and other external causes, which raises the question of whether repeated SAs are not being prevented because of inadequate treatment. ${ }^{74}$ Several studies have been conducted to address the risk population for new SAs, ${ }^{75,76}$ but interventions for SB in adolescents are not well established, which undermines best-practice efforts in this area. Most studies have small sample sizes, and there are several gaps in the evaluation of interventions for SB in young people with identifiable psychopathology. ${ }^{75}$

Several limitations of this review should be considered. Despite the considerable number of articles collected, we must acknowledge that they varied considerably in their methodology, concepts, and measures of treatment compliance, length of follow-up, sample size, instruments utilized to measure outcomes, and main outcomes observed. In addition, studies using multivariate models or adjusting for confounding factors were the exception, leading to further interpretation biases.

Considering the three decades of articles included in this review, important changes to societal or clinical care contexts must be taken into account, as they might affect the phenomenon of treatment compliance in patients with SB. Furthermore, most of the studies were not conducted in countries that offer free access to care, an important limitation regarding treatment compliance. However, interestingly, even with such diverse methodologies, several risk and protective factors were still associated with treatment compliance.

In conclusion, despite poor treatment compliance immediately after the beginning of SB, patients with SB are described as frequent users of MHS. Family factors (such as engaging in more activities and spending more time with parents) and mental health factors (depression, anxiety disorder, borderline personality disorder, and history of a prior $\mathrm{SA}$ ) were found to be related to 
increased MHS utilization. As found for MHS utilization, adolescents with more severe psychopathology were more compliant with treatment. Additionally, prescription of psychotropic medications and interventions in ED and during initial inpatient treatment were shown to improve treatment compliance. Regarding the association between treatment compliance and reattempts, we found no evidence in our review that treatment compliance could reduce the number of reattempts over time.

More studies addressing treatment compliance, focusing on family, ED, and initial inpatient treatment are necessary to better refine interventions that could improve treatment adherence and suicide reattempts in adolescents.

\section{Disclosure}

The authors report no conflicts of interest.

\section{References}

1 McKinnon B, Gariepy G, Sentenac M, Elgar FJ. Adolescent suicidal behaviours in 32 low- and middle-income countries. Bull World Health Organ. 2016;94:340-50F.

2 Jackson CA, Henderson M, Frank JW, Haw SJ. An overview of prevention of multiple risk behaviour in adolescence and young adulthood. J Public Health (Oxf). 2012;34Suppl 1:i31-40.

3 Kann L, McManus T, Harris WA, Shanklin SL, Flint KH, Queen B, et al. Youth risk behavior surveillance - United States, 2017. MMWR Surveill Summ. 2018;67:1-114.

4 Turecki G, Brent DA. Suicide and suicidal behaviour. Lancet. 2016; 387:1227-39.

5 McMahon EM, Corcoran P, Keeley H, Cannon M, Carli V, Wasserman $\mathrm{C}$, et al. Mental health difficulties and suicidal behaviours among young migrants: multicentre study of European adolescents. BJPsych Open. 2017;3:291-9.

6 World Health Organization (WHO). Preventing suicide: a global imperative. Geneva: WHO; 2014.

7 Crosby AE, Han B, Ortega LA, Parks SE, Gfroerer J, Centers for Disease Control Prevention. Suicidal thoughts and behaviors among adults aged $>/=18$ years--United States, 2008-2009. MMWR Surveill Summ. 2011;60:1-22.

8 Dervic K, Brent DA, Oquendo MA. Completed suicide in childhood. Psychiatr Clin North Am. 2008;31:271-91.

9 Goldman-Mellor SJ, Caspi A, Harrington H, Hogan S, Nada-Raja S, Poulton R, et al. Suicide attempt in young people: a signal for long-term health care and social needs. JAMA Psychiatry. 2014;71: 119-27.

10 Shepard DS, Gurewich D, Lwin AK, Reed GA, Jr., Silverman MM. Suicide and suicidal attempts in the United States: costs and policy implications. Suicide Life Threat Behav. 2015;46:352-62.

11 Burstein B, Agostino H, Greenfield B. Suicidal attempts and ideation among children and adolescents in US Emergency Departments, 2007-2015. JAMA Pediatr. 2019;173:598-600.

12 Centers for Disease Control and Prevention (CDC), National Centers for Injury Prevention and Control Web-based Injury Statistics Query and Reporting System. Leading causes of death reports, 1981-2017 [Internet]. 2018 [cited 2020 Sep 23]. webappa.cdc.gov/sasweb/ncipc/ leadcause.html

13 Yoshimasu K, Kiyohara C, Miyashita K, Stress Research Group of the Japanese Society for Hygiene. Suicidal risk factors and completed suicide: meta-analyses based on psychological autopsy studies. Environ Health Prev Med. 2008;13:243-56.

14 Sarchiapone M. Risk and protective factors associated with attitudes toward suicide. Suicidol Online. 2015;6:II-III.

15 Hacker K, Drainoni ML. Mental health and illness in Boston's children and adolescents: one city's experience and its implications for mental health policy makers. Public Health Rep. 2001;116:317-26.

16 Spirito A, Boergers J, Donaldson D. Adolescent suicide attempters: post-attempt course and implications for treatment. Clin Psychol Psychother. 2000;7:161-73.
17 Chan LF, Shamsul AS, Maniam T. Are predictors of future suicide attempts and the transition from suicidal ideation to suicide attempts shared or distinct: a 12-month prospective study among patients with depressive disorders. Psychiatry Res. 2014;220:867-73.

18 Irigoyen M, Porras-Segovia A, Galvan L, Puigdevall M, Giner L, De Leon $\mathrm{S}$, et al. Predictors of re-attempt in a cohort of suicide attempters: a survival analysis. J Affect Disord. 2019;247:20-8.

19 Callor WB, Petersen E, Gray D, Grey T, Lamoreaux T, Bennett PJ. Preliminary findings of noncompliance with psychotropic medication and prevalence of methamphetamine intoxication associated with suicide completion. Crisis. 2005;26:78-84.

20 Pillay AL, Wassenaar DR. Psychological intervention, spontaneous remission, hopelessness, and psychiatric disturbance in adolescent parasuicides. Suicide Life Threat Behav. 1995;25:386-92.

21 Kampman O, Lehtinen K. Compliance in psychoses. Acta Psychiatr Scand. 1999;100:167-75.

22 Sackett DL, Haynes RB. Compliance with therapeutic regimens. Baltimore: Johns Hopkins University Press; 1976.

23 Lizardi D, Stanley B. Treatment engagement: a neglected aspect in the psychiatric care of suicidal patients. Psychiatr Serv. 2010;61:1183-91.

24 Haw C, Houston K, Townsend E, Hawton K. Deliberate self harm patients with depressive disorders: treatment and outcome. J Affect Disord. 2002;70:57-65.

25 Granboulan V, Roudot-Thoraval F, Lemerle S, Alvin P. Predictive factors of post-discharge follow-up care among adolescent suicide attempters. Acta Psychiatr Scand. 2001;104:31-6.

26 Merikangas KR, He JP, Burstein M, Swendsen J, Avenevoli S, Case B, et al. Service utilization for lifetime mental disorders in U.S. adolescents: results of the National Comorbidity Survey-Adolescent Supplement (NCS-A). J Am Acad Child Adolesc Psychiatry. 2011;50:32-45.

27 Borges G, Benjet C, Medina-Mora ME, Orozco R, Familiar I, Nock MK, et al. Service use among Mexico City adolescents with suicidality. J Affect Disord. 2010;120:32-9.

28 Freedenthal S. Racial disparities in mental health service use by adolescents who thought about or attempted suicide. Suicide Life Threat Behav. 2007;37:22-34.

29 Nock MK, Green JG, Hwang I, McLaughlin KA, Sampson NA, Zaslavsky AM, et al. Prevalence, correlates, and treatment of lifetime suicidal behavior among adolescents: results from the National Comorbidity Survey Replication Adolescent Supplement. JAMA Psychiatry. 2013;70:300-10.

30 Renaud J, Berlim MT, Seguin M, McGirr A, Tousignant M, Turecki G. Recent and lifetime utilization of health care services by children and adolescent suicide victims: a case-control study. J Affect Disord. 2009;117:168-73.

31 Springer A, Parcel G, Baumler E, Ross M. Supportive social relationships and adolescent health risk behavior among secondary school students in El Salvador. Soc Sci Med. 2006;62:1628-40.

32 Wozencraft T, Wagner W, Pellegrin A. Depression and suicidal ideation in sexually abused children. Child Abuse Negl. 1991;15:505-11.

33 Wu P, Katic BJ, Liu X, Fan B, Fuller CJ. Mental health service use among suicidal adolescents: findings from a U.S. national community survey. Psychiatr Serv. 2010;61:17-24.

34 Zaborskis A, Sirvyte D, Zemaitiene N. Prevalence and familial predictors of suicidal behaviour among adolescents in Lithuania: a crosssectional survey 2014. BMC Public Health. 2016;16:554.

35 Alvarez BAK. Suicidality among Latina adolescents: the relative effects of psychosocial risk factors and psychological symptoms [dissertation]. Austin: University of Texas; 2014.

36 Burgess S, Hawton K, Loveday G. Adolescents who take overdoses: outcome in terms of changes in psychopathology and the adolescents' attitudes to care and to their overdose. J Adolesc. 1998;21: 209-18.

37 Burns CD, Cortell R, Wagner BM. Treatment compliance in adolescents after attempted suicide: a 2-year follow-up study. J Am Acad Child Adolesc Psychiatry. 2008;47:948-57.

38 Carrizales ID. Loneliness, violence, aggression, and suicidality in incarcerated youth. Stillwater: Oklahoma State University; 2007.

39 Czyz EK, King CA, Nahum-Shani I. Ecological assessment of daily suicidal thoughts and attempts among suicidal teens after psychiatric hospitalization: lessons about feasibility and acceptability. Psychiatry Res. 2018;267:566-74.

40 Giraud P, Fortanier C, Fabre G, Ghariani J, Guillermain Y, Rouviere $\mathrm{N}$, et al. [Suicide attempts by young adolescents: epidemiological 
characteristics of 517 15-year-old or younger adolescents admitted in French emergency departments]. Arch Pediatr. 2013;20:608-15.

41 Groholt B, Ekeberg O. Prognosis after adolescent suicide attempt: mental health, psychiatric treatment, and suicide attempts in a nineyear follow-up study. Suicide Life Threat Behav. 2009;39:125-36.

42 Grupp-Phelan J, McGuire L, Husky MM, Olfson M. A randomized controlled trial to engage in care of adolescent emergency department patients with mental health problems that increase suicide risk. Pediatr Emerg Care. 2012;28:1263-8.

43 Husky MM, Olfson M, He JP, Nock MK, Swanson SA, Merikangas KR. Twelve-month suicidal symptoms and use of services among adolescents: results from the National Comorbidity Survey. Psychiatr Serv. 2012;63:989-96

44 Kataoka S, Stein BD, Nadeem E, Wong M. Who gets care? Mental health service use following a school-based suicide prevention program. J Am Acad Child Adolesc Psychiatry. 2007;46:1341-8.

45 King CA, Segal H, Kaminski K, Naylor MW, Ghaziuddin N, Radpour L. A prospective study of adolescent suicidal behavior following hospitalization. Suicide Life Threat Behav. 1995;25:327-38.

46 King CA, Hovey JD, Brand E, Wilson R, Ghaziuddin N. Suicidal adolescents after hospitalization: parent and family impacts on treatment follow-through. J Am Acad Child Adolesc Psychiatry. 1997;36:85-93.

47 O'Mara RM. Six year outcomes of suicidal adolescents: the role of sexual abuse. A dissertation submitted in partial fulfillment of the requirements for the degree of doctor of philosophy. Michigan: University of Michigan; 2010.

48 Ramchand R, Griffin BA, Harris KM, McCaffrey DF, Morral AR. A prospective investigation of suicide ideation, attempts, and use of mental health service among adolescents in substance abuse treatment. Psychol Addict Behav. 2008;22:524-32.

49 Rotheram-Borus MJ, Piacentini J, Van Rossem R, Graae F, Cantwell C, Castro-Blanco D, et al. Enhancing treatment adherence with a specialized emergency room program for adolescent suicide attempters. J Am Acad Child Adolesc Psychiatry. 1996;35:654-63.

50 Rotheram-Borus MJ, Piacentini J, Van Rossem R, Graae F, Cantwell C, Castro-Blanco D, et al. Treatment adherence among Latina female adolescent suicide attempters. Suicide Life Threat Behav. 1999; 29:319-31.

51 Spirito A, Boergers J, Donaldson D, Bishop D, Lewander W. An intervention trial to improve adherence to community treatment by adolescents after a suicide attempt. J Am Acad Child Adolesc Psychiatry. 2002;41:435-42.

52 Trautman PD, Stewart N, Morishima A. Are adolescent suicide attempters noncompliant with outpatient care? J Am Acad Child Adolesc Psychiatry. 1993;32:89-94.

53 Wharff EA, Ginnis KB, Ross AM, White EM, White MT, Forbes PW. Family-based crisis intervention with suicidal adolescents: a randomized clinical trial. Pediatr Emerg Care. 2019;35:170-5.

54 Yen S, Fuller AK, Solomon J, Spirito A. Follow-up treatment utilization by hospitalized suicidal adolescents. J Psychiatr Pract. 2014;20:353-62.

55 Yen S, Ranney ML, Tezanos KM, Chuong A, Kahler CW, Solomon JB, et al. Skills to enhance positivity in suicidal adolescents: results from an open development trial. Behav Modif. 2019;43:202-21.

56 Spirito A, Lewander WJ, Levy S, Kurkjian J, Fritz G. Emergency department assessment of adolescent suicide attempters: factors related to short-term follow-up outcome. Pediatr Emerg Care. 1994;10:6-12.

57 Normand D, Colin S, Gaboulaud V, Baubet T, Taieb O. How to stay in touch with adolescents and young adults after a suicide attempt? Implementation of a 4-phones-calls procedure over 1 year after discharge from hospital, in a Parisian suburb. Encephale. 2018;44:301-7.

58 DiMatteo MR. Variations in patients' adherence to medical recommendations: a quantitative review of 50 years of research. Med Care. 2004;42:200-9.
59 Hage A, Weymann L, Bliznak L, Marker V, Mechler K, Dittmann RW. Non-adherence to psychotropic medication among adolescents a systematic review of the literature. Z Kinder Jugendpsychiatr Psychother. 2018;46:69-78.

60 Beautrais AL, Joyce PR, Mulder RT. Psychiatric contacts among youths aged 13 through 24 years who have made serious suicide attempts. J Am Acad Child Adolesc Psychiatry. 1998;37:504-11.

61 Gagne S, Vasiliadis HM, Preville M. Gender differences in general and specialty outpatient mental health service use for depression. BMC Psychiatry. 2014;14:135.

62 Smith KL, Matheson FI, Moineddin R, Dunn JR, Lu H, Cairney J, et al. Gender differences in mental health service utilization among respondents reporting depression in a national health survey. Health. 2013;5:1561-71.

63 Vasiliadis HM, Lesage A, Adair C, Boyer R. Service use for mental health reasons: cross-provincial differences in rates, determinants, and equity of access. Can J Psychiatry. 2005;50:614-9.

64 Wang PS, Lane M, Olfson M, Pincus HA, Wells KB, Kessler RC. Twelve-month use of mental health services in the United States: results from the national comorbidity survey replication. Arch Gen Psychiatry. 2005;62:629-40.

65 Hunt IM, Robinson J, Bickley H, Meehan J, Parsons R, McCann K, et al. Suicides in ethnic minorities within 12 months of contact with mental health services. National clinical survey. $\mathrm{Br} \mathrm{J}$ Psychiatry. 2003;183:155-60.

66 Jon-Ubabuco N, Champion JD. Perceived mental healthcare barriers and health-seeking behavior of African-American caregivers of adolescents with mental health disorders. Issues Ment Health Nurs. 2019;40:585-92.

67 Ahern S, Burke LA, McElroy B, Corcoran P, McMahon EM, Keeley H, et al. A cost-effectiveness analysis of school-based suicide prevention programmes. Eur Child Adolesc Psychiatry. 2018;27: 1295-304.

68 Kinchin I, Doran CM. The cost of youth suicide in Australia. Int $\mathrm{J}$ Environ Res Public Health. 2018;15:672

69 Latimer EA, Gariepy G, Greenfield B. Cost-effectiveness of a rapid response team intervention for suicidal youth presenting at an emergency department. Can J Psychiatry. 2014;59:310-8.

70 Swanton R, Collin P, Burns J, Sorensen I. Engaging, understanding and including young people in the provision of mental health services. Int J Adolesc Med Health. 2007;19:325-32.

71 Spirito A. Improving treatment compliance among adolescent suicide attempters. Crisis. 1996;17:152-4.

72 Bushnell GA, Brookhart MA, Gaynes BN, Compton SN, Dusetzina $\mathrm{SB}$, Sturmer T. Examining parental medication adherence as a predictor of child medication adherence in pediatric anxiety disorders. Med Care. 2018;56:510-9.

73 Lachal J, Grandclerc S, Spodenkiewicz M, Moro MR. [Methods to improve suicidal adolescents' compliance to care after emergency discharge: a literature review]. Encephale. 2018;44:465-70.

74 Birkbak J, Stuart EA, Lind BD, Qin P, Stenager E, Larsen KJ, et al. Psychosocial therapy and causes of death after deliberate self-harm: a register-based, nationwide multicentre study using propensity score matching. Psychol Med. 2016;46:3419-27.

75 De Silva S, Parker A, Purcell R, Callahan P, Liu P, Hetrick S Mapping the evidence of prevention and intervention studies for suicidal and self-harming behaviors in young people. Crisis. 2013;34: 223-32.

76 Messiah A, Notredame CE, Demarty AL, Duhem S, Vaiva G, AlgoS investigators. Combining green cards, telephone calls and postcards into an intervention algorithm to reduce suicide reattempt (AlgoS): $\mathrm{P}$-hoc analyses of an inconclusive randomized controlled trial PLoS One. 2019;14:e0210778. 